\title{
EXTINCTIONS AND DISTANCES TO DARK CLOUDS FROM 2MASS, MEGACAM AND IPHAS SURVEYS: LDN 1525 IN THE DIRECTION OF THE AUR OB1 ASSOCIATION
}

\author{
V. Straižys ${ }^{1}$, J. E. Drew ${ }^{2}$ and V. Laugalys ${ }^{1}$ \\ 1 Institute of Theoretical Physics and Astronomy, Vilnius University, \\ Goštauto 12, Vilnius LT-01108, Lithuania; vytautas.straizys@tfai.vu.lt; \\ vygandas.laugalys@tfai.vu.lt \\ 2 Centre for Astrophysics Research, University of Hertfordshire, College Lane \\ Campus, Hatfield, AL109AB, U.K.; j.drew@herts.ac.uk
}

Received: 2010 September 15; revised: November 29; accepted: December 20

\begin{abstract}
The possibility of applying photometry from the 2MASS $J, H, K_{s}$, MegaCam $u, g$ and IPHAS $r, i$, H $\alpha$ surveys for determining the distance to the dark cloud LDN 1525 (TGU 1192) in the direction of the Aur OB1 association is investigated using the red clump giants. The main dust cloud, probably related to the emission nebulae Sh 2-232, Sh 2-233, Sh 2-235, the molecular cloud and the association Aur OB2, is found to be located at a distance of $1.3 \mathrm{kpc}$ from the Sun. The nebula Sh 2-231 can be an object of the Perseus arm. The maximum extinction $A_{V}$ found in the cloud is close to $6 \mathrm{mag}$.
\end{abstract}

Key words: ISM: dust clouds: individual (LDN 1525, TGU 1192) - stars: fundamental parameters (spectral classes, colours) - photometric systems: 2MASS, MegaCam, IPHAS

\section{INTRODUCTION}

In a previous paper (Straižys \& Laugalys 2009, hereafter Paper I) we investigated the possibility of applying photometry from the 2MASS $J, H, K_{s}$, MegaCam $u, g$ and IPHAS $r, i$ surveys for the isolation of the red clump giants (hereafter RCGs) with a small admixture of G5-K1 and M2-M3 stars of the giant branch. RCGs then can be applied for the determination of interstellar reddenings and distances to dust clouds. These stars are extremely useful for the investigation of extinction up to great distances, since their absolute magnitudes in the $K_{s}$ passband, $M_{K_{s}}=-1.6$, exhibit only a rather small dispersion (Alves 2000; Grocholski \& Sarajedini 2002). According to Straižys \& Lazauskaitè (2009), the mean intrinsic colour indices of RCGs are: $J-H=0.46$ and $H-K_{s}=0.09$.

In Paper I, in order to isolate RCGs we used the stars falling within certain limits of the interstellar reddening-free photometric parameters $Q_{J H K_{s}}$ and $Q_{u g r i}$ and of the $J-H$ and $H-K_{s}$ colour indices. The method was applied to the dark cloud LDN 935 separating the North America and Pelican nebulae in Cygnus.

In the present paper we extend the application of the method to another Milky 
Way area in the direction of the Aur OB1 association, close to the Galactic anticenter. The choice of the area was predetermined by the availability in it of $u$, $g, r, i$ photometry obtained with the MegaCam mosaic CCD camera on CFHT (Gwyn 2008; MegaPipe 2009) and of the unpublished IPHAS $r$, i, H $\alpha$ photometry.

\section{THE AREA IN AURIGA}

The selected $1^{\circ} \times 1^{\circ}$ area in the direction of the Aur OB1 association with the center at $\mathrm{RA}(2000)=05^{\mathrm{h}} 39^{\mathrm{m}} 13^{\mathrm{s}}, \operatorname{DEC}(2000)=+35^{\circ} 46^{\prime} 05^{\prime \prime},\left(\ell=173.478^{\circ}, b=\right.$ $+2.448^{\circ}$ ) is shown in Figure 1. It covers a part of the dust cloud LDN 1525 (Lynds 1962) = TGU 1192 (Dobashi et al. 2005), with the emission nebulae Sh 2-231, Sh 2-232, Sh 2-233 and Sh 2-235 (Sharpless 1959). The largest nebula, Sh 2-232, enters the area only partly.

The listed nebulae are considered to belong to a giant molecular cloud (Heyer et al. 1996) related to the Aur OB1 association, which covers an area of $8^{\circ} \times 12^{\circ}$ and is located at a distance of $1.32 \mathrm{kpc}$ from the Sun (Humphreys 1978). Most recently the region was described in detail by Reipurth \& Yan (2008). The brightest nebula, Sh 2-235, is known to be excited by the star BD+35 1201 (O9.5, $V=10.54)$, the nebula Sh 2-232 by HD 37737 (O9.5 or B0 star, $V=8.06$ ) and the nebula Sh 2-231 by ALS $8476(\mathrm{O} 9 \mathrm{~V}, V=10.79)$. Hunter \& Massey (1990) write that the nebula Sh 2-233 is excited by a B1.5 II star, but their identification chart marks the object in the center of the nebula which, according to other investigations, is a compact H II region, probably coinciding with the IRAS $05351+3549$ object.

Since the absolute magnitudes of the exciting stars are known with low accuracy, spectroscopic distances of the nebulae are very uncertain (between 1.0 and $2.3 \mathrm{kpc}$, see Heyer et al. 1996). Most of the authors accept a mean distance of 1.8 kpc. However, this distance places the cloud in the interarm region between the Local and the Perseus arms, what seems to be unrealistic. The kinematical method, based on the Galactic rotation curve, is not applicable in this region which is close to the anticenter, where radial velocities due to the circular rotation of the disk are close to zero. Therefore, any new determination of the distance to the complex is important.

There is a problem with the identification of the Sh 2-233 nebula (see Israel \& Felli 1978). Its position given by Sharpless (1959) falls in an empty area located about $4^{\prime}$ below a bright compact emission object of $\sim 2^{\prime}$ diameter seen at RA (2000) $=05: 38: 32, \operatorname{DEC}(\mathrm{J} 2000)=+35: 51: 20$, which is not present in the catalog. Its diameter coincides with that given by Sharpless to the object in the "empty area". There is no doubt that the coordinates of Sh 2-233 in the Sharpless catalog are in error. Therefore we will assume (together with Israel \& Felli 1978) that the object at $05: 38: 32,+35: 51: 20$ is Sh 2-233. At $6.5^{\prime}$ east of this "empty area" a double cluster of infrared objects has been found and named Sh 2-233IR (IRAS 05358+3543, see Reipurth \& Yan 2008; Ginsburg et al. 2009; Yan et al. 2010).

The area is surrounded by the large molecular cloud G173.7+02.7 $\left(104^{\prime} \times 80^{\prime}\right)$ investigated in the molecular radio lines of CO by Evans \& Blair (1981), Dame et al. (1987, 2001), Heyer et al. (1996) and Kawamura et al. (1998). Its mean radial velocity $V_{\mathrm{LSR}}=-16.6 \mathrm{~km} / \mathrm{s}$ (Kawamura et al. 1998). The cloud covers a large part of the area investigated in this paper, except of its lower right quarter. The dust distribution map from Schlegel et al. (1998) taken from the Internet's Virtual Telescope SkyView is shown in Figure 2. Here again the lower right part of the area gives evidence of a lower emission intensity, i.e., lower dust density. The 


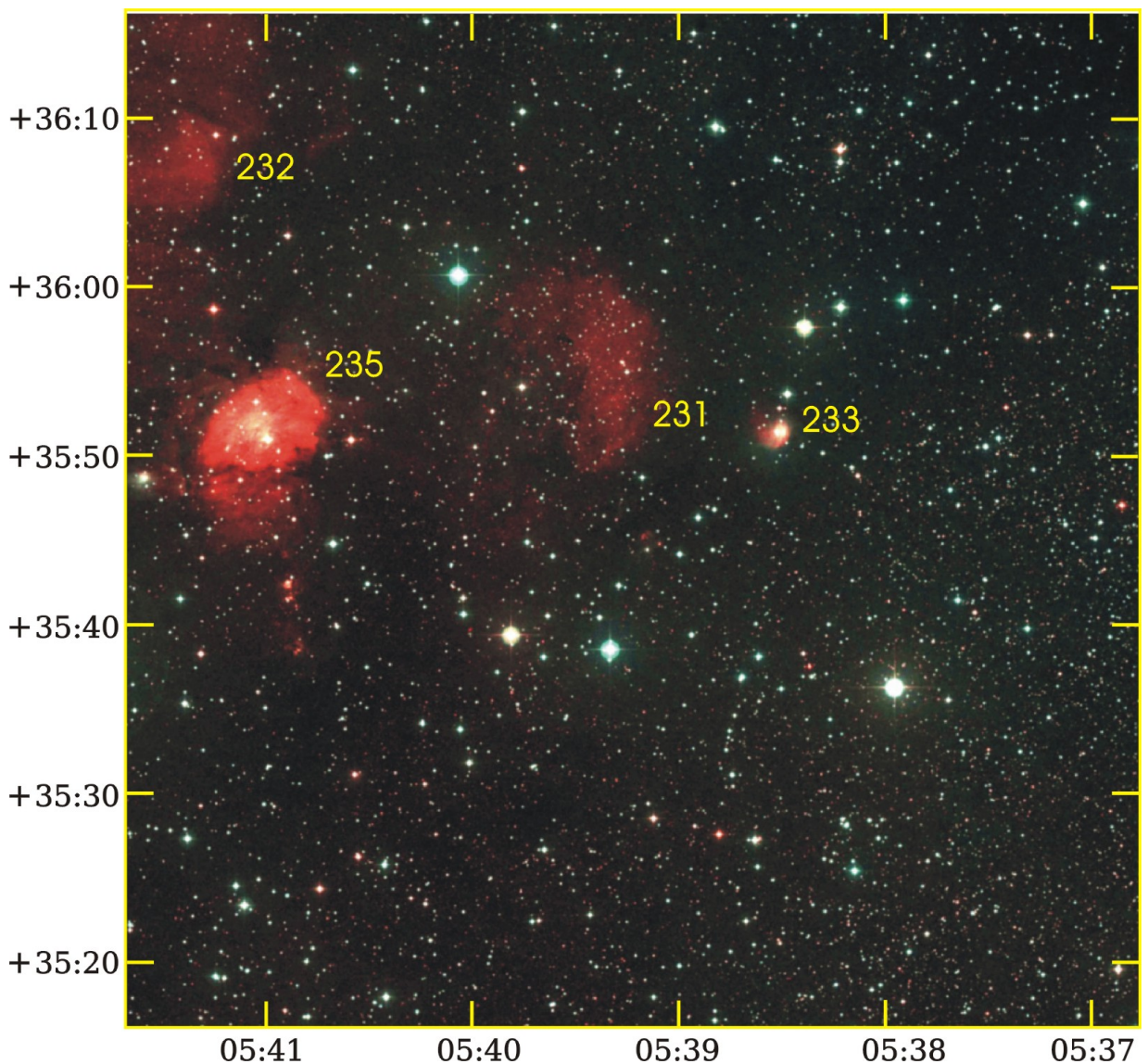

Fig. 1. The investigated area in the Aur OB1 association. The numerals denote the emission nebulae Sh 2-231, Sh 2-232, Sh 2-233 and Sh 2-235. The colour picture is composed from the blue and red images of the DSS2 survey.

extinction distribution in the area from the Dobashi et al. (2005) atlas is shown in Figure 3.

\section{THE $\mathrm{u}-\mathrm{g}$ vs. $\mathrm{r}-\mathrm{i}$ DIAGRAM}

Intrinsic colour indices in the MegaCam $u-g$ system and IPHAS $r-i$ system for the main-sequence stars and red giants were calculated by synthetic photometry, as it was described in Paper I. For $u$ and $g$ passbands the MegaCam filter transmittance and the quantum efficiency functions were taken from the MegaCam Internet site. ${ }^{1}$ The transmittance functions of the filters $r$ and $i$ and the quantum efficiency function of CCD were taken from the INT WFC and IPHAS Internet sites. $^{2}$ For synthetic photometry, the spectral energy distributions of stars from Straižys \& Sviderskienè (1972) and Sviderskienè (1988, 1992) were taken.

\footnotetext{
${ }^{1}$ http://www2.cadc-ccda.hia-iha.nrc-cnrc.gc.ca/megapipe/docs/filters.html

${ }^{2}$ http://www.iphas.org and http://www.ing.iac.es/Astronomy/instruments/wfc
} 


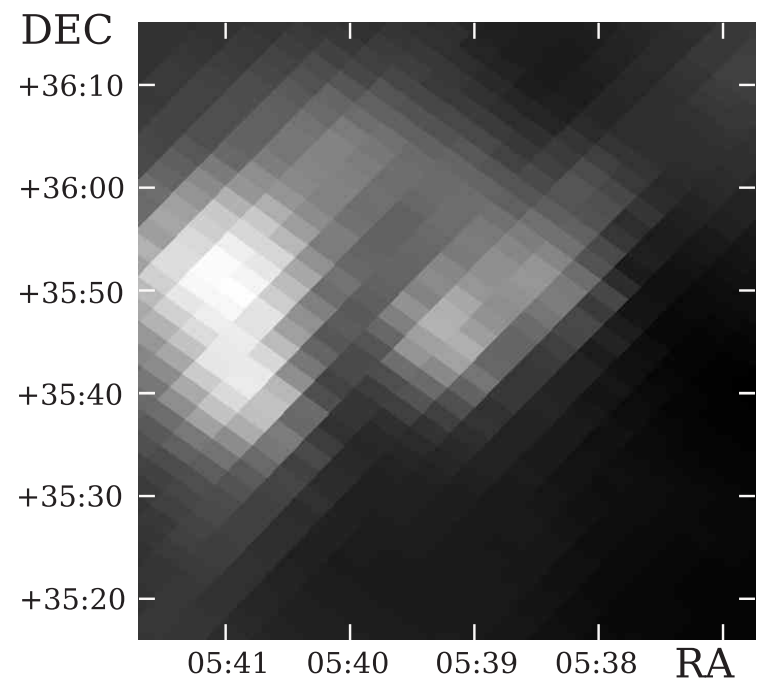

Fig. 2. The investigated area in the dust distribution map from Schlegel et al. (1998).

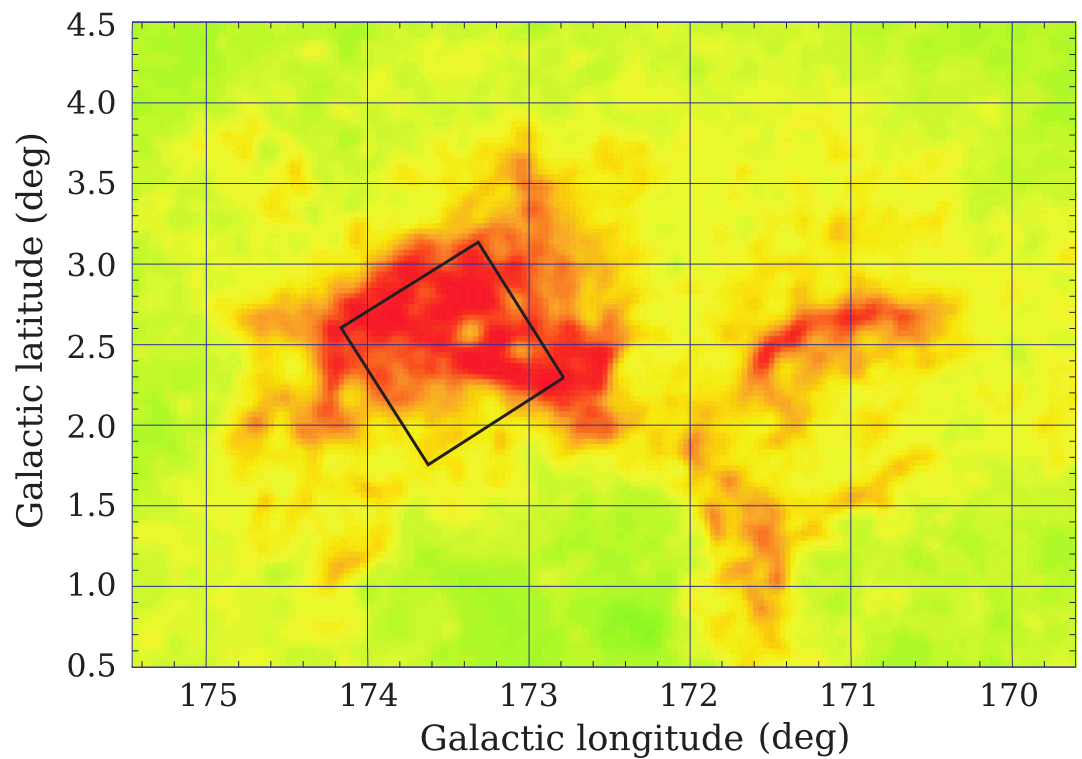

Fig. 3. The investigated area (in the black rectangular) in the Dobashi et al. (2005) atlas of dark clouds.

Since the observed MegaCam magnitudes are in the AB magnitude system, and our synthetic colour indices are normalized to zero for an A0 V star (Vega), we have corrected the synthetic indices $u-g$ by adding 0.44 mag, this constant being taken from the MegaCam Internet site. The IPHAS $r-i$ values are always normalized to zero for an A0 V star (Drew et al. 2005). The accepted values of the intrinsic colour indices $u-g$ and $r-i$ are listed in Table 1 of Paper I. The slopes 


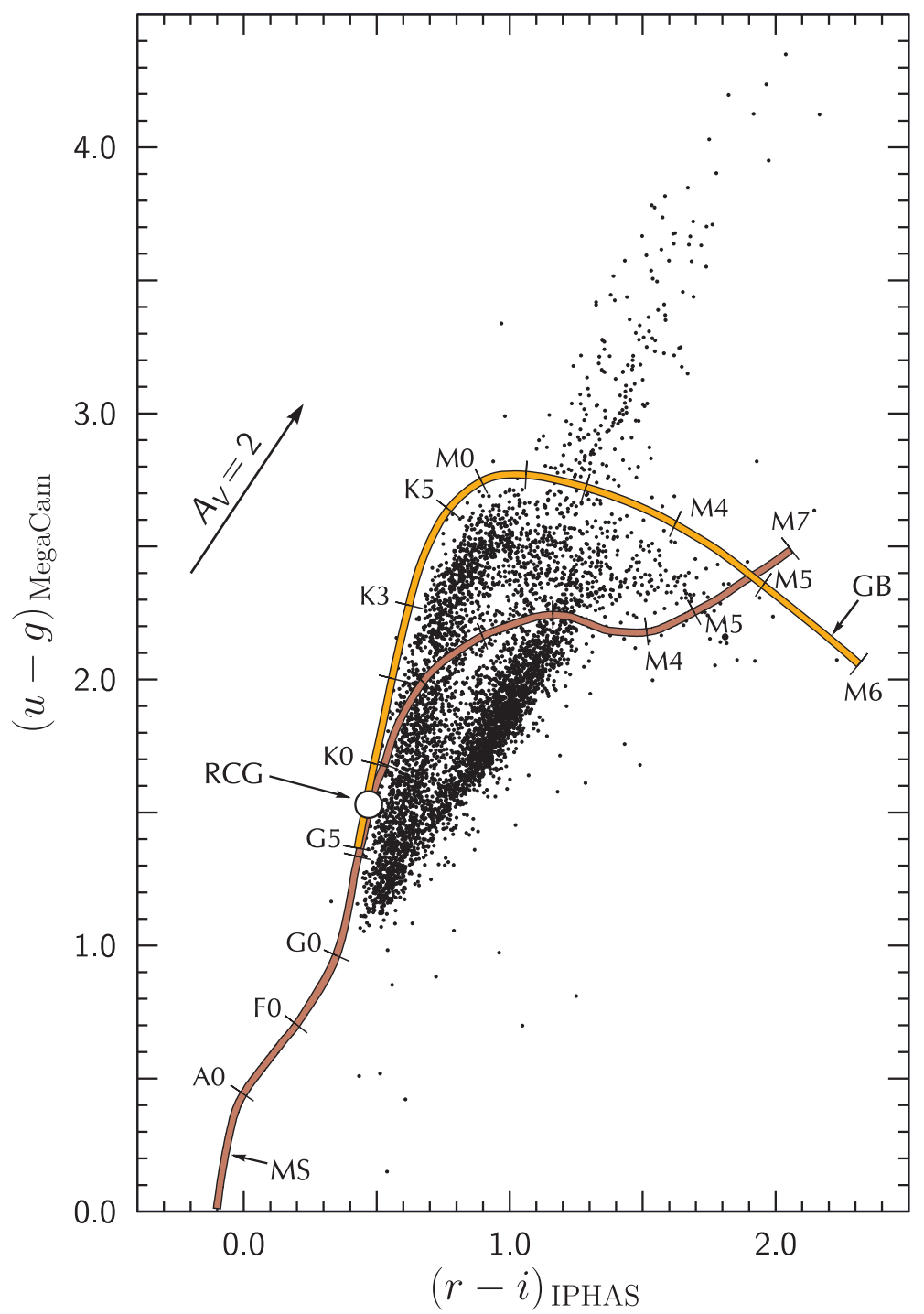

Fig. 4. Observed stars in the $u-g$ vs. $r-i$ diagram. Color indices $u-g$ are from MegaCam and $r-i$ are from IPHAS. Only the stars with the magnitude errors $\leq 0.05$ mag are plotted.

of the reddening lines in the $u-g$ vs. $r-i$ diagram depend slightly on spectral type - for RCGs and early K-giants these are close to 1.50. The schematic diagram and the description of the sequences are given in Paper I.

The Auriga MegaCam field also contains the $r$ and $i$ data. However, the images of the brightest stars in the survey are saturated and have large systematic errors. In the Auriga field the saturated magnitudes are for stars with $u<14.1, g<15.4$, $r<14.9$ and $i<14.7$. Therefore, we used only the MegaCam $u$ and $g$ magnitudes fainter than 14.1 and 15.4, respectively. The MegaCam magnitudes $r$ and $i$ were replaced with the corresponding IPHAS magnitudes which are not saturated up 
to magnitude 13 .

In the Auriga field we identified about 5150 stars having unsaturated measurements in the MegaCam $u$ and $g$ passbands and in the IPHAS $r$ and $i$ passbands to an accuracy limit of 0.05 mag. The stars are plotted in Figure 4, together with the intrinsic lines of the main sequence and red giants from Paper I. The distribution of stars in this diagram is the combined result of the real distribution of stars on the intrinsic sequences of different luminosity classes, different shifts of stars along their reddening lines, and the limiting magnitudes both at the bright and faint ends of different passbands.

The two most distinguishing features in the $u-g$ vs. $r-i$ diagram are the well populated sequence of $\mathrm{G}-\mathrm{K}$ stars of different luminosities (the left-hand sequence) and the sequence of reddened $\mathrm{A}-\mathrm{F}$ stars of luminosities $\mathrm{V}-\mathrm{III}$ (the right-hand sequence with $r-i$ between 0.5 and 1.3). The absence of stars on and close to the intrinsic sequences is the result of the brightest limiting magnitudes described above. This effect excludes stars with low reddening (and extinction) since they are too bright at least in one of the four passbands.

\section{2MASS J-H vs. $\mathrm{H}-\mathrm{K}_{s}$ DIAGRAM AND THE RED CLUMP GIANTS}

The $J-H$ vs. $H-K_{s}$ diagram for 1790 2MASS stars, common with MegaCam, is plotted in Figure 5. Only the stars with the errors of all magnitudes $\leq 0.05$ were considered. The intrinsic sequences of luminosity $\mathrm{V}$ and III and the reddening line are from Figure 2 of Paper I. The $J-H$ vs. $H-K_{s}$ diagram alone does not allow us to isolate RCGs in the presence of interstellar reddening since they cover the main sequence in the G2-M2 range and the giant branch in the G5-M5 range (see Straižys \& Lazauskaite 2009 and Paper I).

In Paper I, for the isolation of RCGs (together with G5-K1 giants on the ascending branch of the HR diagram), we adopted several conditions reducing the number of overlapping stars from other sequences. In the present paper, these conditions for the $u-g$ vs. $r-i$ diagram were slightly modified.

1. In the $J-H$ vs. $H-K_{s}$ diagram the requirement remains the same as in Paper I: the star must lie inside the grey strip shown in Figure 2 of Paper I, outlined by two reddening lines with $Q_{J H K_{s}}=0.18$ and 0.38 .

2 . In the $u-g$ vs. $r-i$ diagram, the requirement was set that the star must lie inside the grey strip shown in Figure 3 of Paper I, outlined by the reddening lines with $Q_{u g r i}$ between 0.68 and 0.98 . This requirement separates RCG stars from giants cooler than K1. Since the strip crosses the sequence of red giants of class M, it is impossible to separate the reddened RCGs from stars of types M2-M3 III.

3. To exclude the main-sequence stars K5 and earlier, we rejected all the stars with $r-i \leq 0.8$. Unfortunately, this condition can also exclude RCGs with small reddenings, if they are not yet diminished by the brightest limiting magnitude $i$.

Applying these conditions, we have selected a sample of 194 stars plotted in Figures 5 and 6 as red crosses. ${ }^{3}$ We consider that most of these stars are RCGs with a small admixture of normal subgiant and giant branch stars of spectral types G5-K1 IV-III and M3 III. The faintest stars of the selected RCGs are of limiting magnitude $g=20$ mag.

For the suspected RCGs the values of interstellar extinction $A_{V}$ and distance

\footnotetext{
${ }^{3}$ The list of the selected RCGs is available from the authors.
} 


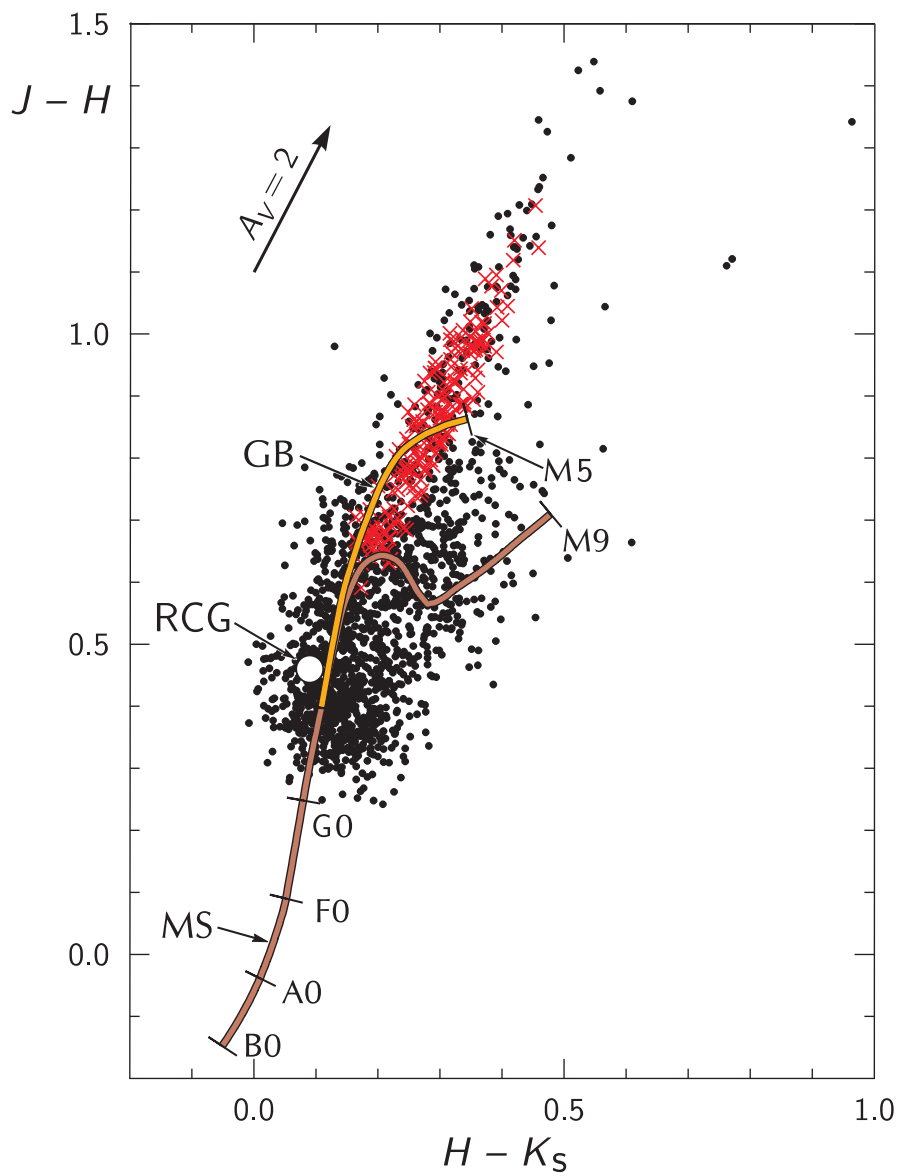

Fig. 5. The $J-H$ vs. $H-K_{s}$ diagram for 2 MASS stars common with MegaCam. Only stars with the magnitude errors $\leq 0.05$ are plotted. The main sequence, giant branch and the locus of red clump giants (white circle) are overplotted from Figure 2 of Paper I. Red crosses denote the suspected RCGs.

$d$ were calculated using the following equations and taking $M_{r}=+0.6$ and $(r-i)_{0}$ $=0.47$ :

$$
\begin{gathered}
A_{r}=4.0\left[(r-i)-(r-i)_{0}\right], \\
5 \log d=r-M_{r}+5-A_{r}, \\
A_{V}=1.20 A_{r} .
\end{gathered}
$$

The ratio $A_{r} / E_{r-i}=4.0$ was calculated by synthetic photometry accepting the normal interstellar extinction law, which for B-A stars gives $A_{V} / E_{B-V}=3.2$.

The extinctions $A_{V}$ for RCGs are plotted against distances in Figure 7 as dots. The most distant suspected RCGs are at $d \approx 8 \mathrm{kpc}$, i.e., farther than the Outer arm. We do not exclude that the stars between 6 and $8 \mathrm{kpc}$ are not RCGs but stars of lower luminosities (probably reddened $\mathrm{G}-\mathrm{K}$ subgiants).

The locus of RCGs in Figure 7 at high values of extinctions is limited by the curve corresponding to the limiting magnitude $g=20$. At low distances and 


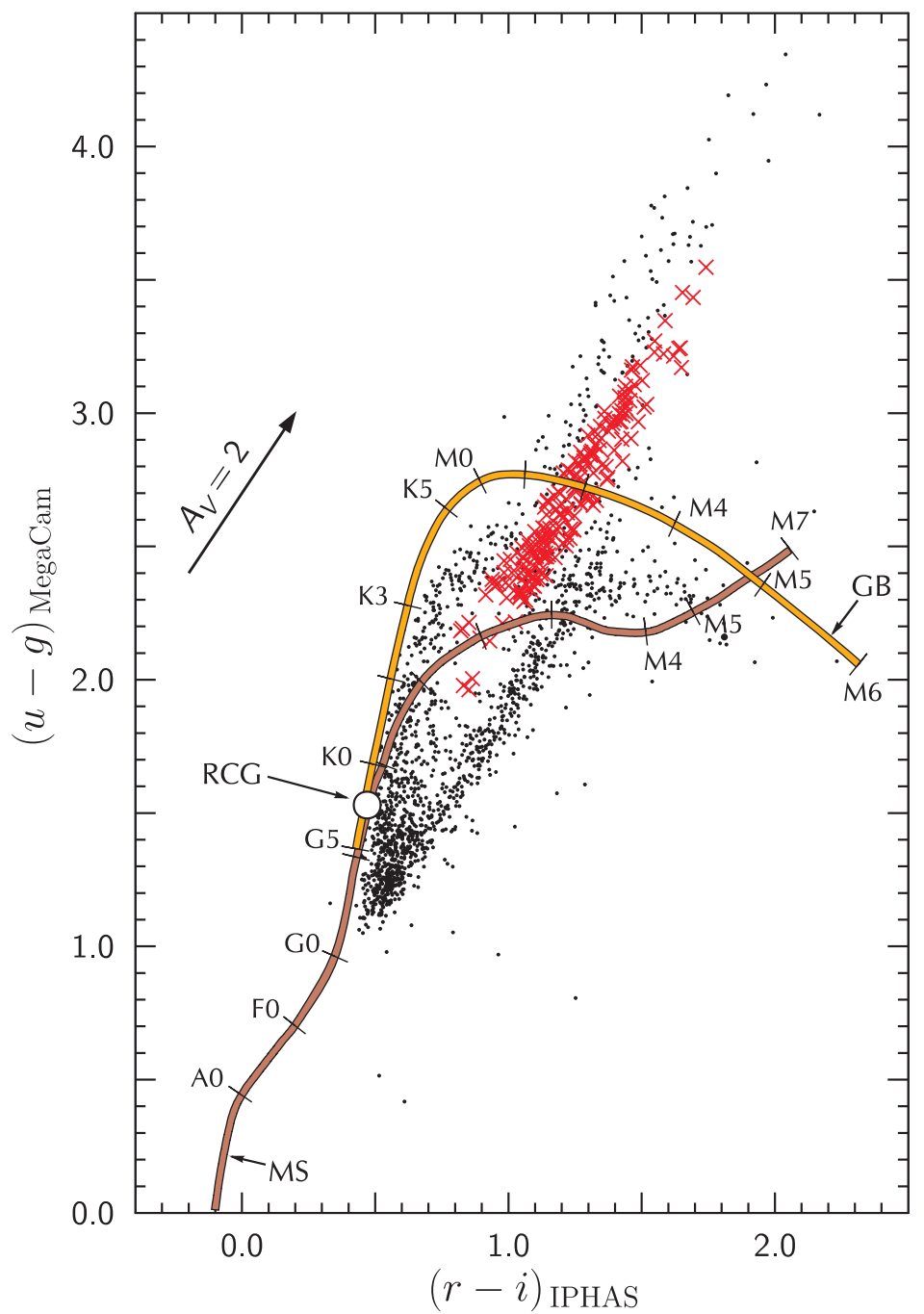

Fig. 6. The $u-g$ vs. $r-i$ diagram for the Auriga area stars which have measurements of the $J, H$ and $K_{s}$ magnitudes in the 2MASS survey. Only the 2MASS, MegaCam ( $u$ and $g$ ) and IPHAS ( $r$ and $i$ ) magnitudes with errors $\leq 0.05$ mag were considered. Red crosses denote the suspected RCGs.

extinctions the set is limited by the curve for $i=13$ : the stars having brighter $i$ magnitudes are absent in the sample due to saturated images.

\section{DISCUSSION AND CONCLUSIONS}

In Figure 8 the surface distribution of the RCGs is shown. The highest density of stars is observed in the direction of the transparent regions - a large region in the lower right corner, a smaller region in the direction of the nebula Sh 2-231 and in a few small regions in the north. All these transparent regions can be easily identified in the Dobashi et al. (2005) map, Figure 3. In the direction of 


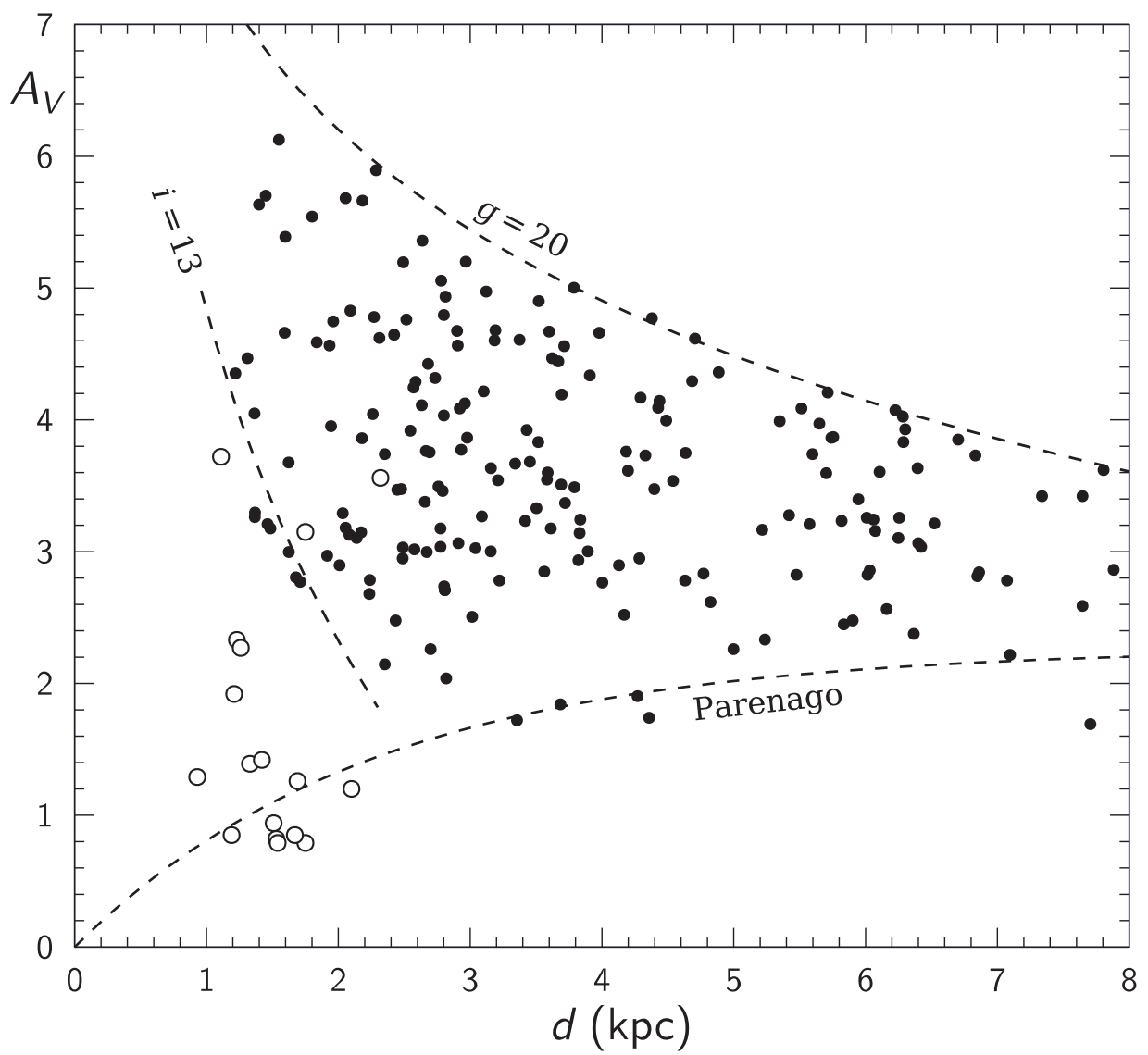

Fig. 7. Interstellar extinction $A_{V}$ as a function of distance in the Auriga area. 194 RCGs are plotted as dots and 17 OB stars from the Aur OB1 association as circles. The limiting magnitude curves are explained in the text. The extinction curve calculated with the exponential Parenago formula is shown.

the dark clouds RCGs are scarce since the foreground stars are mostly too bright (saturation), and the background stars are too faint (extinction) to be represented in the sample.

The $A_{V}$ vs. $d$ diagram shows that the heavily reddened RCGs are apparently farther than $1.2 \mathrm{kpc}$. The statistics of RCGs in the Hipparcos catalog (Paczyński \& Stanek 1998) suggests that in the Auriga area of $1^{\circ} \times 1^{\circ}$ size the number of expected RCGs up to $1 \mathrm{kpc}$ distance is about 20. Most of them are probably excluded from our sample due to their brightness in the $i$ passband which saturates CCD images at long exposures; see the curve in Figure 7 corresponding to $i=13$ mag, the limiting magnitude of the brightest RCGs. Consequently, only the stars with $A_{V}$ between 4 and 6 mag can be used for the estimation of the distance to the front edge of the dust cloud.

The Catalog of Stars in Stellar Associations and Young Clusters by Humphreys \& McElroy (1984) lists 36 members of the association Aur OB1. Only a few of them fall in the investigated area. In Figure 7 we plot 17 stars of spectral classes 


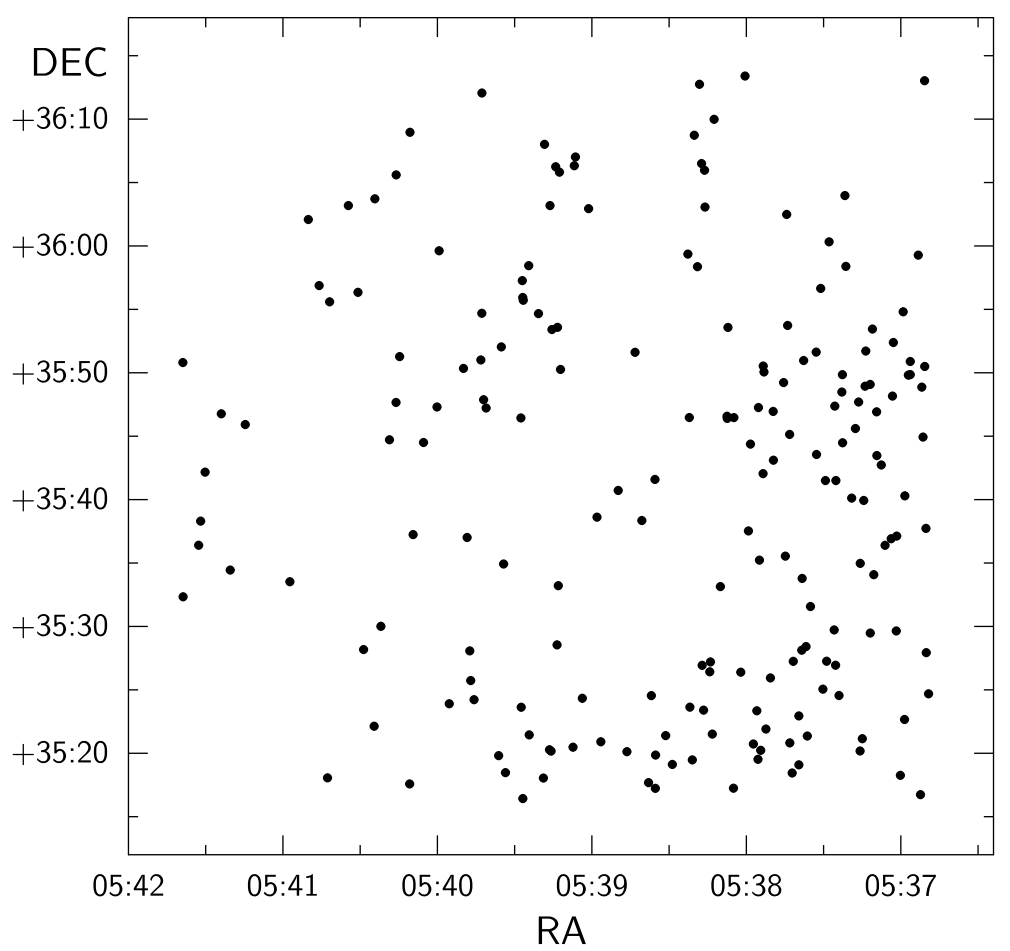

Fig. 8. Surface distribution of the identified RCGs in the investigated area.

O7-B2 which are considered to be the members of the association and are located within $\sim 2^{\circ}$ from the center of the investigated area. Their spectral classes and $B, V$ magnitudes are taken from the Simbad database, intrinsic colours and absolute magnitudes from Straižys (1992). Most of these stars in the diagram (open circles) fall in the distance range between 1 and $2 \mathrm{kpc}$. Since the absolute magnitudes of O-B2 stars can be estimated only from their MK types, distances to the potential members of the association are of low accuracy: both spectral and luminosity classes estimated in different literature sources sometimes are considerably different. The calibration of luminosity classes in absolute magnitudes is also quite uncertain. The best object for the estimation of the distance to Aur OB1 is the open cluster M36 = NGC 1960 which is considered to be the association center. According to extensive photometric and kinematic study by Sanner et al. (2000), the distance to M36 is $1.3 \mathrm{kpc}$. This value is in agreement with the dust cloud distance estimated for RCGs in the present paper.

It is interesting to verify distances to the stars responsible for the ionization of emission nebulae in the investigated area. For the calculation of extinctions and distances to these stars we used spectral types and $B, V$ magnitudes from Georgelin et al. (1973).

The exciting star of the nebula Sh 2-235, BD +35 1201 (O9.5 V, $V=10.54)$, shows the largest extinction $\left(A_{V}=3.7\right)$ among the Aur OB1 members and its photometric distance is found to be $1.1 \mathrm{kpc}$. The exciting star of Sh 2-232, HD 37737 (B0 V, $V=8.07$ ), is a spectral binary and its distance is uncertain. For the exciting star of Sh 2-231, LS V +35 $24=$ ALS 8476 (O9 V, $V=10.79, A_{V}=3.6$ ), 
we obtain a distance of $2.3 \mathrm{kpc}$, and this places it in the Perseus arm. The region of the Sh 2-231 nebula contains many RCGs seen at large distances. Also, this region coincides with a 'gulf' in the molecular cloud G173.7+02.7 what is consistent with a low density of the interstellar gas (Kawamura et al. 1998). All this mean that in the direction of Sh 2-231 we have a semitransparent window in which the objects of the Perseus arm are seen. Inside the nebula Sh 2-233 Hunter \& Massey (1990) have identified a star of spectral type B1.5 II and found its distance $4.7 \mathrm{kpc}$. However, this distance is of rather low accuracy since the magnitude $V$ and colour index $B-V$ of this star were estimated from spectroscopy rather than photometry. A large scatter in distances found for the nebulae may mean that it is premature to relate all of them to the same star forming region.

In Figure 7 we also plotted the curve of interstellar extinction $A_{V}$ according to the exponential formula proposed by Parenago (1945) and calibrated by Sharov (1963). It is evident that the Parenago curve, calculated for the Galactic latitude $b=+2.5^{\circ}$ with the coefficients $A_{0}=1 \mathrm{mag} / \mathrm{kpc}$ and $\beta=0.1 \mathrm{kpc}$, runs along the lower boundary of both star samples (RCGs and OB stars), as it is expected for the general Galactic dust layer in the directions free of dense clouds.

The above discussion leads to the conclusion that the distance to the dust clouds in the area is $1.2-1.3 \mathrm{kpc}$, what is consistent with the distances to the Aur OB1 association and the cluster M36. The emission nebulae Sh 2-232, Sh 2-233, Sh 2-235 and the molecular cloud belong probably to the same complex. However, the nebula Sh 2-231 can be an object of the Perseus arm seen through a semitransparent window. The maximum extinction $A_{V}$ found in the cloud is $\sim 6$ mag but this does not mean that in some other directions it cannot be much larger. In front of the cloud the extinction is of the order of $1 \mathrm{mag}$, and this is in agreement with the exponential Parenago law. The distance to the dust cloud places it near the outer edge of the Local arm.

ACKNOWLEDGMENTS. The use of the 2MASS, MegaPipe, IPHAS, SkyView, Gator and Simbad databases is acknowledged. We are thankful to Romualda Lazauskaite for calculating synthetic interstellar extinctions and to Edmundas Meištas and Stanislava Bartašiūte for their help in preparing the paper.

\section{REFERENCES}

Alves D. R. 2000, ApJ, 539, 732

Dame T. M., Hartmann D., Thaddeus P. 2001, ApJ, 547, 792

Dame T. M., Ungerechts H., Cohen R. S. et al. 1987, ApJ, 322, 706

Dobashi K., Uehara H., Kandori R. et al. 2005, PASJ, 57, S1

Drew J. E., Greimel R., Irwin M. J. et al. 2005, MNRAS, 362, 753

Evans N. J., Blair G. N. 1981, ApJ, 246, 394

Georgelin Y. M., Georgelin Y. P., Roux S. 1973, A\&A, 25, 337

Ginsburg A. G., Bally J., Yan C.-H., Williams J. P. 2009, ApJ, 707, 310

Grocholski A. J., Sarajedini A. 2002, AJ, 123, 1603

Gwyn S. D. J. 2008, PASP, 120, 212

Heyer M. H., Carpenter J. M., Ladd E. F. 1996, ApJ, 463, 630

Humphreys R. M. 1978, ApJS, 38, 309

Humphreys R. M., McElroy D. B. 1984, Catalogue of Stars in Stellar Associations and Young Clusters, Univ. of Minnesota, CDS Strasbourg Catalogue V/44 
Hunter D. A., Massey P. 1990, AJ, 99, 846

Israel F. P., Felli M. 1978, A\&A, 63, 325

Kawamura A., Onishi T., Yonekura Y., Dobashi K. et al. 1998, ApJS, 117, 387

Lynds B. T. 1962, ApJS, 7, 1

MegaPipe 2009, http://www2.cadc-ccda.hia-iha.nrc-cnrc.gc.ca/megapipe

Paczyński B., Stanek K. Z. 1998, ApJ, 494, L219

Parenago P. P. 1945, AZh, 22, 129

Perryman M. A. C., Brown A. G. A., Lebreton Y. et al. 1998, A\&A, 331, 81

Reipurth B., Yan C.-H. 2008, in Handbook of Star Forming Regions, vol. 1, ASP, p. 869

Sanner J., Altmann M., Brunzerdorf J., Geffert M. 2000, A\&A, 357, 471

Schlegel D. J., Finkbeiner D. P., Davis M. 1998, ApJ, 500, 525

Sharov A. S. 1963, AZh, 40, $900=$ Soviet Astronomy, 7, 689

Sharpless S. 1959, ApJS, 41, 257

Straižys V. 1992, Multicolor Stellar Photometry, Pachart Publishing House,

Tucson, Arizona; available in pdf format from

http://www.itpa.lt/MulticolorStellarPhotometry/

Straižys V., Laugalys V. 2009, Baltic Astronomy, 18, 141 (Paper I)

Straižys V., Lazauskaitè R. 2009, Baltic Astronomy, 18, 19

Straižys V., Sviderskienė Z. 1972, Bull. Vilnius Obs., No. 35, 3

Sviderskienè Z. 1988, Bull. Vilnius Obs., No. 80, 3

Sviderskienè Z. 1992, Bull. Vilnius Obs., No. 86, 3

van Leeuwen F. 2007, A\&A, 474, 653; CDS Catalog No. I/311

Yan C.-H., Minh Y. C., Wang S.-Y. et al. 2010, ApJ, 720, 1 